\title{
Research on Fractal Features in Milling ZrO2 Ceramics
}

\author{
Jianxun Xue ${ }^{*}, 1,2$, Lulin $\mathrm{An}^{1}$ and Quanping $\operatorname{Sun}^{2}$ \\ ${ }^{I}$ College of Mechanical and Electrical Engineering, Nanjing University of Aeronautics and Astronautics, Nanjing, \\ Jiangsu, 210016, P.R. China \\ ${ }^{2}$ Lab of Advanced Manufacturing Technology, Huaiyin Institute of Technology, Huaian, Jiangsu, 223003, P.R. China
}

\begin{abstract}
In order to study the fractal features of the tool profile of worn surface and cutting force during the process of cutting hard brittle materials such as ceramics, the milling experiments of $\mathrm{ZrO} 2$ were carried out. The images of worn surface and cutting force were collected and preprocessed with digital image processing technology and phase space reconstruction method. Then the fractal dimensions were calculated and the change regularity was analyzed. The research findings show that the fractal dimension of worn surface is affected by wear rates and machining time, and can be different while the wear loss is same. The fractal dimension of cutting force oscillates with a high-low-high general trend.
\end{abstract}

Keywords: Capacity dimension, correlation dimension, tool wear, zirconia.

\section{INTRODUCTION}

Zirconia ceramic is a widely-used typical ceramic material with such characteristics as heat resistance, wear resistance, corrosion resistance etc. Compared with the metallic material, the completely sintered zirconia ceramic has a high hardness allowing for quick wear during cutting thus influencing the appearance quality and dimensional accuracy of the workpieces. Workpieces may be scrapped when the tool is severely worn. Therefore, it is necessary to study and monitor the tool wear during this process.

A great deal of research has indicated that tool wear is characterized by irregularity, nonlinearity, and randomness. The indicators such as common average wear width $V B$, maximum wear width $V B_{\max }$ etc. contain limited information content and are not able to characterize the random behaviors and the detail characteristics of the tool wear. As an important branch of the nonlinear science, the fractal theory provides a quantitative description method for studying the high irregularities in the natural world. Fractal originally means irregularities, fragments etc. Fractal dimension is fundamental quantity used to characterize the complexity and the self-similarity of an object in the fractal theory [1]. The larger the fractal dimension, the higher the complexity and the irregularity of the research object, vice versa.

Some scholars have applied the fractal theory to research on tool wear. Such research has focused on fractal during the process of metallic material cutting. There is no research on fractal during the process of cutting of hard and brittle materials. From the perspective of the research method, the majority of the research involves calculating the fractal dimension of these signals and indirectly reflecting the degree of irregularity of tool wear with workpiece surface roughness [2-4], acoustic emission [5-7], vibration [8-10] etc. being the objects. There is little fractal research using digital image directly obtained from the tool wear zone. Using fractal dimension to directly characterize the irregularity of the tool wear profile requires optical instruments for imaging. It is susceptible to the working environment but it has an intuitive result and definite physical significance.

The paper applies the fractal theory to research on tool wear in milling of zirconia ceramic, analyzes the fractal characteristics of the tool wear profile and cutting force, and expects to provide a foundation for optimized cutting parameters of the hard and brittle materials and on-line monitoring of tool wear.

\section{EXPERIMENTS}

Experimental Conditions: The samples were completely sintered zirconia ceramics with a dimension of $52 \mathrm{~mm} \times 24 \mathrm{~mm} \times 13 \mathrm{~mm}$; the tool used the Sweden Sandvik clip-type single-blade PCD tool, model R390-11T304E-P4NL CD10; machine tool model Mikron UCP800 Duro; those images of the tool wear zone were obtained with the help of the OLYMPUS DSX100 microscope; the cutting force was measured with a Kistler 9257B dynamometer and its corresponding Kistler 5070 charge amplifier. The experimental setup is as shown in Fig. (1).

Two groups of milling parameters. Group A: Spindle speed $n=2000 \mathrm{r} / \mathrm{min}$, Feed Speed $v_{f}=60 \mathrm{~mm} / \mathrm{min}$, Axial Cutting Depth $a_{p}=3 \mathrm{~mm}$, Radial Cutting Depth $a_{e}=0.1 \mathrm{~mm}$, without addition of cutting fluid. Group B: Spindle speed $n=1500 \mathrm{r} / \mathrm{min}$, Feed Speed $v_{f}=60 \mathrm{~mm} / \mathrm{min}$, Axial Cutting Depth $a_{p}=3 \mathrm{~mm}$, Radial Cutting Depth $a_{e}=0.06 \mathrm{~mm}$, without addition of cutting fluid. 


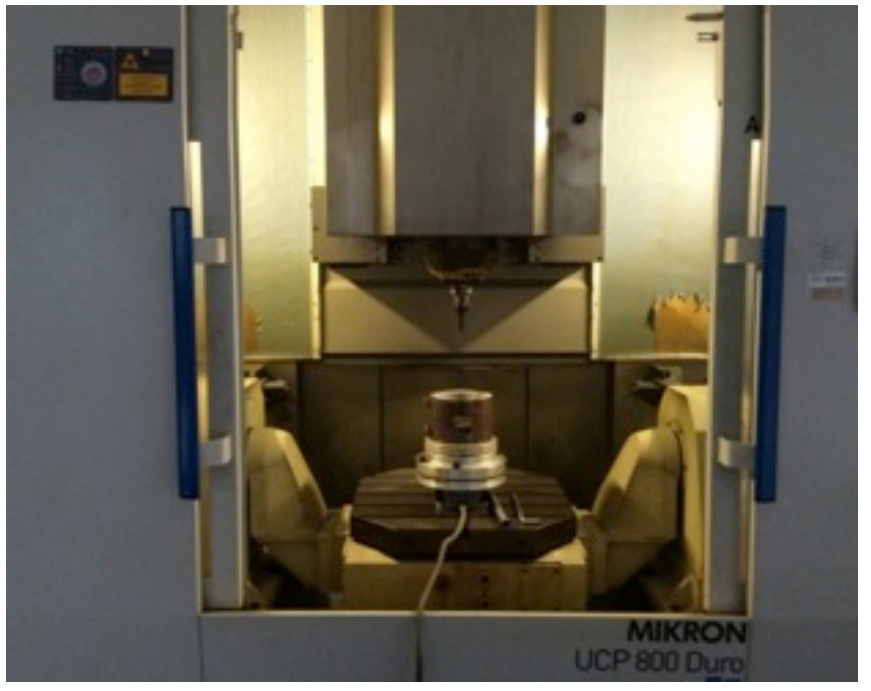

Fig. (1). Experimental set-up.

The single cutting length for each group of parameters was $24 \mathrm{~mm}$ and the tool flank surface image was collected once every time when the sample was cut 10 times (cutting time: $4 \mathrm{~min}$ ). The cutting force was measured each time and the cutting force was measured 80 times in total for each group of parameters. The direction of the cutting force was defined as: cutting direction $\mathrm{X}$, feed direction $\mathrm{Y}$, axial direction of tool $\mathrm{Z}$.

\section{FRACTAL RESEARCH ON TOOL WEAR PROFILE}

Zirconia ceramic was highly brittle. The tool wear resulting from the milling processing of zirconia ceramic was concentrated on the tool flank surface. Thus the tool flank surface exhibited a significant irregularity as shown in Fig. (2).

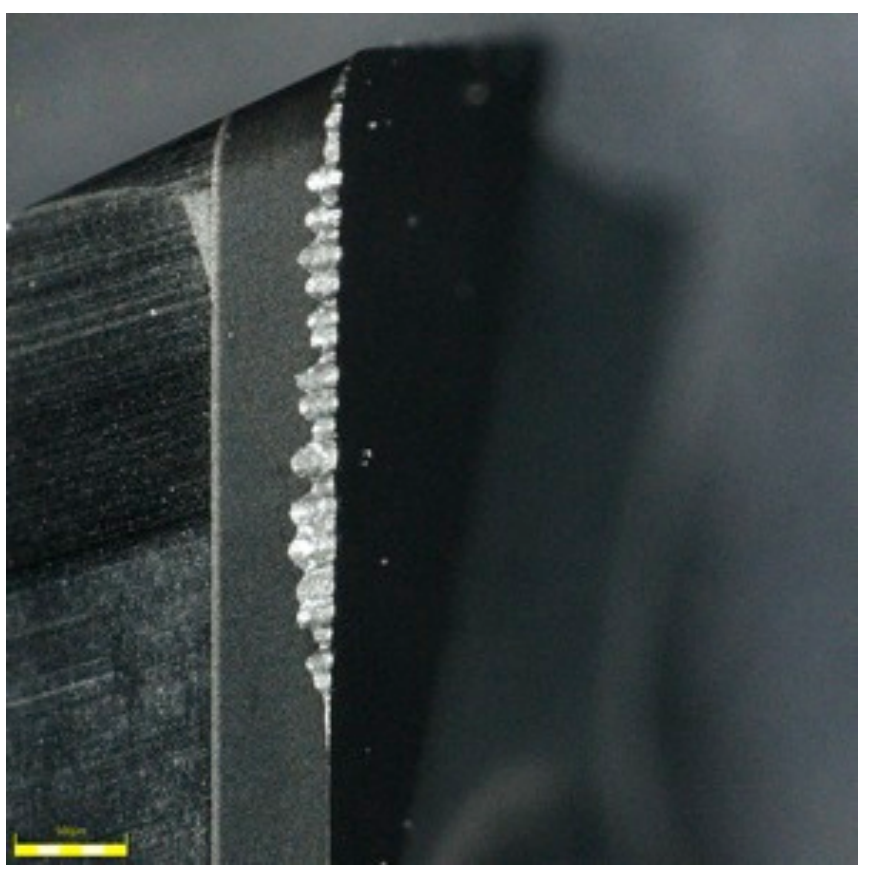

Fig. (2). Tool Flank Surface (cutting time $\mathrm{t}=32 \mathrm{~min}$, Group A).

\subsection{Extraction of Wear Profile}

In Reference [11], the wear zone was subjected to imaging, sampling, and analog-digital conversion to form a 512X512 dot matrix image for the tool wear zone through an optical microscope. The paper used a different digital picture processing technique to extract the wear profile: first, the BMP image collected was converted into a grayscale image; then, the image was preprocessed to reduce the noise and increase the contrast; the image was segmented with the Canny operator; finally, the image of the left profile of the wear band was obtained through morphological dilation and corrosion operations.

\subsection{Calculation of Tool Wear Capacity}

A line was drawn perpendicular to the right profile from a point of the left profile of the wear band to obtain the perpendicular in pixel. The wear width of the point was calculated by multiplying the perpendicular length by $3.2 \mu \mathrm{m}$, the length represented by each pixel. All pixel points on the wear profile were subjected to the above operations. The average value obtained was the average wear width $V B$. The accuracy of the result was significantly higher than that of the result obtained by selecting only several points. Fig. (4) presents the curve for the average wear width $V B$ varying with the cutting time. As seen in the Fig. (3), the wear process was divided into initial wear, normal wear, and sharp wear and normal wear stage of Group B was significantly longer than that of Group A.

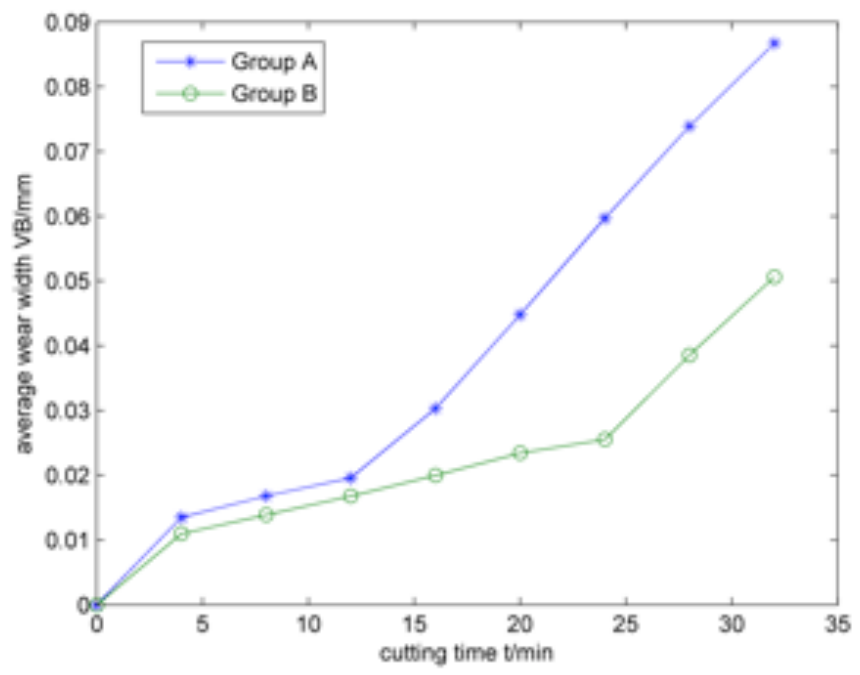

Fig. (3). Variation curve for average wear width.

\subsection{Calculation of Fractal Dimension}

Consideration must be given to presence or absence of fractal characteristics of the tool wear profile during calculation of the fractal dimension. Self-similarity was the basic characteristic of fractal. Any small parts should be similar to the whole. There was little self-similarity in a strict sense. Most of the fractal was self-similarity in a statistical sense. It met the requirements within a certain size range. 
The box-counting dimension is a common practical method for calculating the graphical fractal dimension. It is applicable to calculations less than two dimensions or in the vicinity of two dimensions. Small boxes with a side length of $\delta$ were taken to cover the figure. The number of non-empty boxes was $\mathrm{N}$. If the scaling relation $N \propto \delta^{-D}$ existed, the fractal existed making calculation of fractal dimension possible. As a common fractal dimension, the capacity dimension $D_{0}$ can be calculated by using the following formula:

$D_{0}=-\lim _{\delta \rightarrow 0} \frac{\ln N}{\ln \delta}$

Generally, a series of $\delta_{i}$ were taken to obtain appropriate $N_{i}$. These points were fitted into a straight line in the double logarithmic plot. The absolute value of its slope was the capacity dimension $D_{0}$.

The double logarithmic plot for the wear profile in Fig. (3) obtained by the box-counting method is as indicated in Fig. (4). $\delta$ was in pixel. It was thus clear that $\ln N$ and $\ln \delta$ exhibited an approximate linear relation within a certain scaling range. It indicated that the tool wear profile had a generalized self-similarity and could be described with fractal dimension.

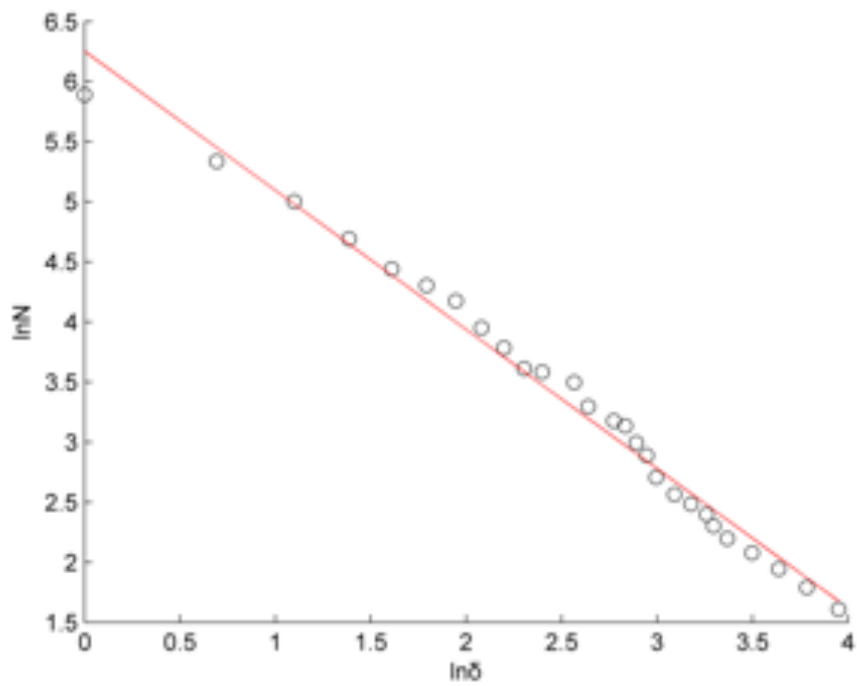

Fig. (4). Double logarithmic plot ( $\mathrm{t}=32 \mathrm{~min}$, group $\mathrm{A})$.

The tool flank wear profiles with different cutting time obtained were analyzed to obtain the curve for capacity dimension $D_{0}$ varying with cutting time as shown in Fig. (5). The tool wear capacity was integrated representation of wear strength and cutting time. A curve of the relationship between capacity dimension $D_{0}$ and tool wear capacity by transformation of coordinates, as shown in Fig. (6). As seen in the figure: (1) the capacity dimension $D_{0}$ of Group A was always larger than that of Group B within the same cutting time. As seen in Fig. (4), the tool wear rate in Group A was significantly higher than that in Group B indicating that the larger the wear rate, the greater the fractal dimension within the same cutting time. In Reference [11], the authors studied the fractal dimension when the 45 \# carbon steel was cut with a high speed steel tool at different cutting speeds. The authors pointed out that the variation of the cutting speed was responsible for different tool wear strengths and fractal dimensions; the larger the wear strength, the greater the fractal dimension; (2)the tool wear curve of each group of parameters approximated a straight line when entering the sharp wear stage indicating that the wear rates were roughly the same within this interval; but the capacity dimension $D_{0}$ was still increasing, that was to say capacity dimensions $D_{0}$ were different under the same wear rate indicating that the wear rate was not the only factor that determined the capacity dimension $D_{0}$ and the cutting time was also an important factor that influenced the capacity dimension $D_{0}$; the capacity dimension $D_{0}$ increased with the cutting time under the same wear rate; (3) the capacity dimension $D_{0}$ of Group A was always larger than that of Group B under the same tool wear capacity. It indicated that the capacity dimensions $D_{0}$ are different under different cutting parameters and the same tool wear capacity.

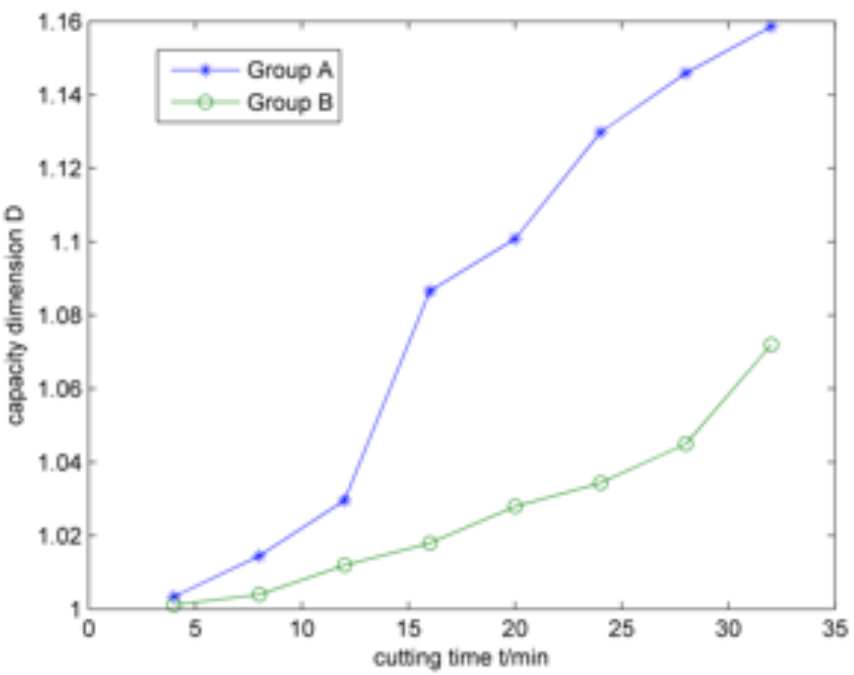

Fig. (5). Curve of relationship between capacity dimension and cutting time.

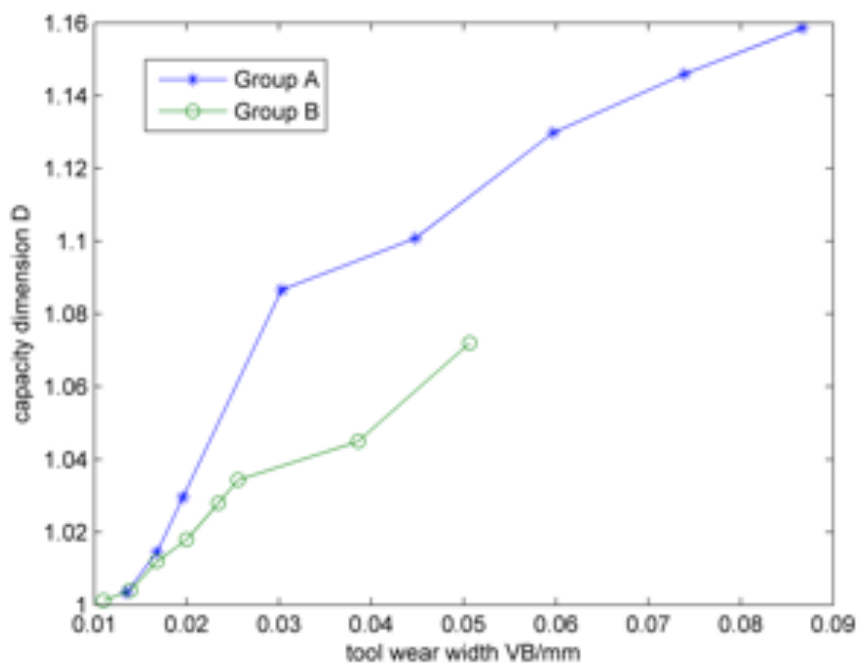

Fig. (6). Curve of relationship between capacity dimension and wear width. 


\subsection{Calculation of Generalized Fractal Dimension}

Capacity dimension $D_{0}$ was a simple fractal dimension. It was generally considered that it was too simple to describe the fractal with only one simple fractal dimension due to the fact that it was not able to completely describe the characteristics and might cause loss of information. Therefore, multi-fractal was needed to be introduced for more detailed description. The generalized fractal dimension $D_{q}$ is an important method for description of multi-fractal. In the box-counting dimension method, the probability of falling into the $i$ th was $P_{i}$. For any parameter $q$, Renyi defined generalized entropy

$K_{q}(\delta)=\frac{\ln \sum_{i=1}^{N} P_{i}^{q}(\delta)}{1-q}$

Accordingly, the generalized dimension can be defined as

$D_{q}=-\lim _{\delta \rightarrow 0} \frac{K_{q}(\delta)}{\ln \delta}$

When $q=0,1,2, \quad D_{q}$ are capacity dimension $D_{0}$, information dimension $D_{1}$, and correlation dimension $D_{2}$ respectively. Fig. (7) presents the curve for three dimensions varying with the cutting time under the parameters of Group A. It can be seen from the figure that the trends of the three dimensions varying with the cutting time completely coincide. It indicated that using the information dimension $D_{1}$ and correlation dimension $D_{2}$ can also obtain the same conclusion compared with using the capacity dimension $D_{0}$.

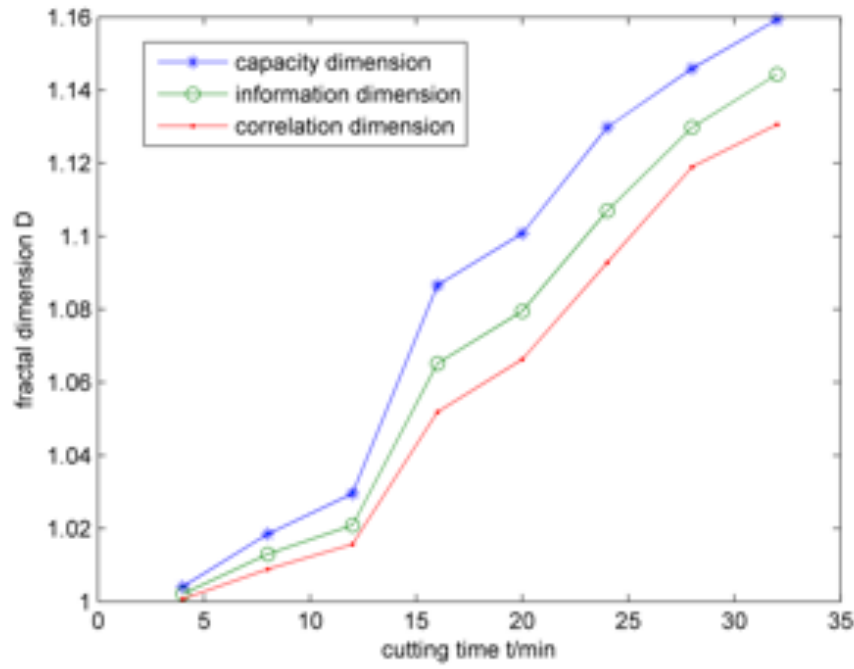

Fig. (7). Curve of Relationship between three dimensions and cutting time (group A).

\section{STUDY ON FRACTAL DIMENSION OF CUTTING FORCE SIGNAL}

Cutting force is an important parameter during cutting. It comprises static component and dynamic component. The static component is the average value of the cutting force. The dynamic component fluctuates above and below the average value. The cutting force signal is correlated with the tool wear. When wear occurs to the tool, the area of contact between the tool and workpiece, frictional force, and dynamic component change [12]. On the contrary, variation in dynamic component does not necessarily result from tool wear. It is an integrated reflection as a result of interactions among various physical behaviors. Much implied information will be lost if the temporal evolution of the cutting force signal is directly analyzed. The temporal evolution $D_{2}$ is a common fractal dimension. It is sensitive to the time and process behavior of the system. It can properly reflect the dynamic characteristics of the system. The theoretical value of the periodic signal correlation dimension $D_{2}$ is 1 . The random signal is in a completely chaotic state with a theoretical value of its correlation dimension $D_{2}$ of $\infty$. The signal between both is a positive fraction [13].

The G-P algorithm proposed by Grassberger and Procaccia in 1983 was a classical method for defining and calculating the correlation dimension. It was also an extremely important breakthrough in research on chaotic time series. Phase space was reconstructed for the onedimension time series with the time difference method. $\mathrm{N} m$ dimensional vectors were obtained. A point $X_{i}$ in the phase space was arbitrarily selected as the reference point. The distances between another $\mathrm{N}-1$ points and the reference point were calculated. We calculated the number of the points falling into a volume element with $X_{i}$ being the center and and the small scalar $r$ being the radius and further obtained the correlation integral $C(r)$ as follows:

$$
C(r)=\frac{1}{N(N-1)} \sum_{i \neq j}\left[H\left(r-\left\|X_{i}-X_{j}\right\|\right)\right] \sqrt{a^{2}+b^{2}}
$$

where, $\mathrm{H}(\cdot)$ is Heaviside step function. When $\mathrm{r} \rightarrow 0$, the correlation dimension $D_{2}$ is as follows:

$D_{2}=\lim _{r \rightarrow 0} \frac{d \ln C(r)}{d \ln r}$

The experiment used the side-edge milling method. The overall radial cutting force was much larger than the tangential force and axial force. Thus, the dynamic component of the radial force was taken for study. During calculation of the correlation dimension $D_{2}$, it was vital to determine the delay time $\tau$ and embedded dimension $m$. The autocorrelation function method and the average mutual information method were two common methods to obtain the delay time $\tau$. The autocorrelation function method only measured the linear dependence of the variable and the average mutual information method considered the nonlinear dependence. Therefore, the paper used the average mutual information method to determine the delay time, $\tau=4$. In term of selection of the embedded dimension $m$, the saturated correlation dimension method was used to obtain $m=12$, which met the embedding theorem. The no-scale interval was determined on the basis of the Richardson trilinear method. For the 80 groups of radial forces collected under the parameters of Group A, the stationary data within 1 second (2000 points) were taken to calculate the correlation dimension and obtain the curve of the relationship between 
the correlation dimension $D_{2}$ of the dynamic component of the cutting force and the cutting time, as shown in Fig. (8).

Compared with the variation curve for the correlation dimension of the cutting force of titanium alloy in Reference [14], it is clear that (1) the correlation dimension $D_{2}$ exhibits a high-low-high variation process with the changes in cutting time on the whole regardless of zirconia ceramic or titanium alloys material. The variation process corresponds to the three stages of tool wear. Both the wear rates at the early and late stages of tool wear are higher than that at the normal stage. (2)A higher wear rate indicates more significant and complex changes in cutting force; the correlation dimension $D_{2}$ of the cutting force of zirconia ceramic is oscillating and the amplitudes are different at all stages; the correlation dimension of the cutting force of titanium alloy has no significant oscillation. As a brittle material, zirconia ceramic is different from metal in cutting. Its cutting process is generally accompanied by initiation and propagation of cracks, formation of chips [15]. The chips are largely irregular fragments at the initial stage of cutting and become more irregular powder with the increasing wear. The irregularity in formation of chips will necessarily cause irregularity in cutting force and also directly influence the correlation dimension of the cutting force.

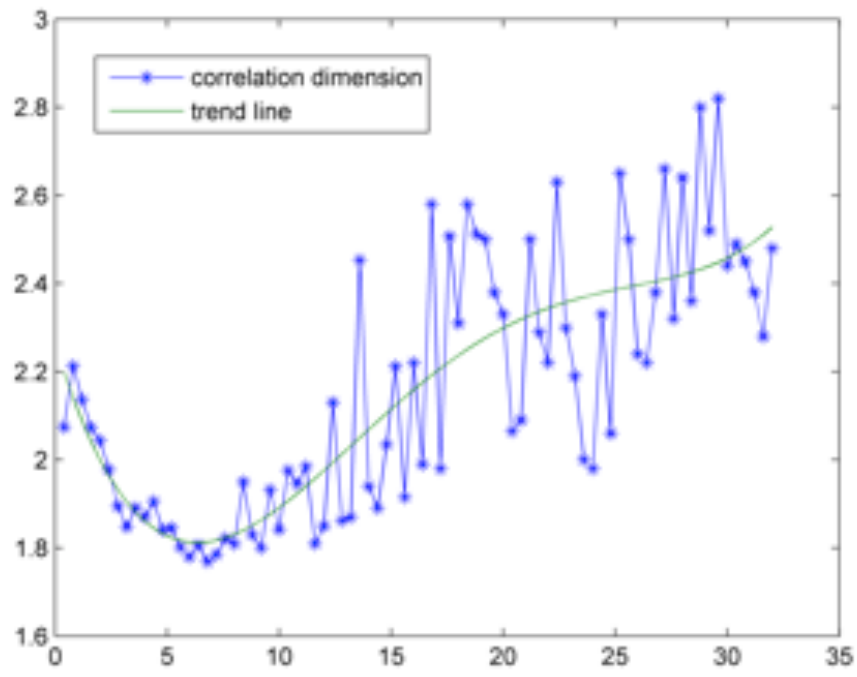

Fig. (8). Curve for relationship between correlation dimension of dynamic component and cutting time.

\section{CONCLUSION}

(1) The fractal dimension of the tool wear profile is correlated with the cutting time in cutting of hard and brittle materials. Within the same time, the higher the wear rate, the larger the fractal dimension. The fractal dimension is increasing with time under the same wear rate.

(2) Selecting proper cutting parameters and decreasing the tool wear rate can not only prolong the tool life but also reduce the irregularity degree of the tool wear profile thus contributing to improvement of the surface quality of workpieces.
The fractal dimension of hard and brittle materials oscillates and exhibits a high-low-high trend on the whole. The trend corresponds to the three stages of tool wear. The trend can be used to monitor the wear condition of tool.

\section{CONFLICT OF INTEREST}

The authors confirm that this article content has no conflict of interest.

\section{ACKNOWLEDGEMENTS}

This work was financially supported by National Natural Science Foundation (51075173) of China, the Open Fund of Jiangsu Key Laboratory of Digital Machining Technology (HGDML-0912) and Young Teacher Foundation of Huaiyin Institute(HGC1001).

\section{REFERENCES}

[1] A. Accardo, M. Affinito, M. Carrozzi and F. Bouquet, "Use of the fractal dimension for the analysis of electroencephalographic time series", Biological Cybernetics, vol. 77, no. 5, pp. 339-350, November 1997.

[2] Bin $\mathrm{Li}$, "On the use of fractal methods for the tool flank wear characterization", International Journal of Refractory Metals and Hard Materials, vol. 42, pp. 221-227, January 2014.

[3] Motoyoshi Hasegawa, Jiancheng Liu, Koichi Okuda and Masayuki Nunobiki, "Calculation of the fractal dimensions of machined surface profiles", Wear, vol. 192, no. 1-2, pp. 40-45, March 1996.

[4] Zhang G and Gopalakrishnan S, "Fractal geometry applied to online monitoring of surface finish", International Journal of Machine Tools and Manufacture, vol. 36, no. 10, pp. 1137-1150, October 1996.

[5] Zhang Kaifeng, Yuan Huiqun and Nie Peng, "Tool wear condition monitoring based on generalized fractal dimensions" , Journal of Vibration and Shock, vol. 33, no. 1, pp. 162-164, 2014.

[6] Govekar E, Gradišek J and Grabec I, "Analysis of acoustic emission signals and monitoring of machining processes", Ultrasonics, vol. 38, no. 1-8, pp. 598-603, March 2000.

[7] Wang Zhongmin, Wang Xinyi, Chen Aidi, Yang Dayong and Jia Yuping. "New method of on-line monitoring tool wear states based on fractal dimension", Journal of Beijing Institute of Technology, vol. 20, no. 4, pp. 441-444, August 2000.

[8] Davies M A and Balachandran B, "Impact dynamics in milling of thin-walled structures", Nonlinear Dynamics, vol. 22, no. 4, pp. 375-392, August 2000

[9] Bukkapatnam S T S, Kumara S R T and Lakhtakia A, "Fractal estimation of flank wear in turning" , Journal of Dynamic Systems Measurement and Control, vol. 122, no. 1, pp. 89-94, March 1999.

[10] Chuangwen $\mathrm{Xu}$, Hualing Cheng and Limei Liu, "The fractal characteristic of vibration signals in different milling tool wear periods", in Seventh International Symposium on Instrumentation and Control Technology, 2008, pp. 712809-712809-6.

[11] Mei Anhua and Wang Jinghui, "The application of fractal theory in the research of cutting tool wear" , Journal of Wuhan Technical University of Surveying and Mapping, vol. 22, no. 4, pp. 66-69, December 1997.

[12] Zhang Jialiang, Li Peizhi and Pang Jingzhu, "Study on cutting force characteristics in tool wear process", Manufacturing Technology and Machine Tool, vol. 16, no. 5, pp. 111-113, May 2010

[13] Jianming. Zheng, "Research on drill wear monitoring technology of the multi-feature fusion based on HMM", Ph.D. dissertation, Xi'an University of Technology, Xi'an, Shanxi, China, 2004. 
[14] Zheng Guangming, Zhao Jun and Wang Xiaoqin, "Study on fractal characterization of cutting force in dry turning Titanium alloy with carbide tool" , Tool Engineering, vol. 44, no. 1, pp. 2325, January 2010.
[15] Ueda K, Sugita T and Tsuwa H, "Application of fracture mechanics in micro-cutting of engineering ceramics" , CIRP Annals-Manufacturing Technology, vol. 32, no. 1, pp. 83-86, 1983.

Received: February 17, 2014

Revised: March 21, 2015

Accepted: June 9, 2015

(C) Xue et al.; Licensee Bentham Open.

This is an open access article licensed under the terms of the (https://creativecommons.org/licenses/by/4.0/legalcode ), which permits unrestricted, non-commercial use, distribution and reproduction in any medium, provided the work is properly cited. 